\title{
PC BASED COMPUTER- AIDED TRAINING PROGRAM FOR HARBOR STRUCTURE DESIGN
}

\author{
$\underline{\text { Yunsu } \mathrm{Na}^{1}}$, Sunghwan Park ${ }^{1}$, Jihyun $\mathrm{Nam}^{2}$, Sangmo $\mathrm{Ahn}^{3}$, Ryo Yamamoto ${ }^{3}$, Jang-Ho Jay Kim ${ }^{1}$, \\ and YunMook Lim ${ }^{1 *}$ \\ ${ }^{1}$ Department of Civil Engineering, Yonsei University, Seoul, Korea \\ ${ }^{2}$ C.E.O, S.E.Nest Co., LTD., Seoul, Korea \\ ${ }^{3}$ Civil Business Unit, Marine \& Port Engineering Team, Samsung C\&T, Seoul, Korea \\ *Corresponding author(yunmook@yonsei.ac.kr)
}

\begin{abstract}
The design engineers for harbor structures normally learned the trades from their company or self-educated at the beginning of their career. Due to this unsystematic education approach, each design company has its own design format and accumulated design databases. Therefore, the objective of this study is to develop a design program for personnel computer usage that enables young engineers to learn about designing of harbor structures such as caisson type of breakwaters. This program uses a unified design format written in MS Visual Basic for the user interface and the MS Excel for the actual design calculation. This program contains well developed GUI including drawings and input values as well as immediate display of calculated values. Moreover, the input values are saved as a text file so that it may be edited for various design conditions and process. Ultimately, this program allows optimization of the total costs among various design conditions. The program will greatly help beginning engineers to understand about the optimum design and overall design procedure.
\end{abstract}

Keywords: Harbor Structure Design, Computer Aided, Caisson, Cost Optimization

\section{INTRODUCTION}

Recently, harbor structures have become an important infrastructure from the perspective of environmental sustainability, safe costal management, and international trade. Due to Kyoto Protocol Agreement, reduction of $\mathrm{CO} 2$ emission and global warming issues has become focal point of construction industry.So, many countries are attempting to construct harborstructures as the international trade volumes are continuously increasing. Presently, the number of harbor construction project iscurrently increasing at a pace the world has never seen before. Internationally and domestically, the harbor structures have become bigger and more complicated. Moreover they are constructed in the deep sea and poor soil condition. Unfortunately, there is currentlyautomated and unified design and construction process for breakwater and quay wall structures construction for fast pace planning. Since the design methods use simplified equations which calculate complex design wave height and pressure or use approximated values of exces- sive design and errors can occur in the design. Also, Korean technologies for manufacturing, launching, and constructing of caisson are at world standard, but design level is far below world standard. In addition, the design of harbor structures is not normally included engineering education in higher education institutes in the world. The beginning design engineers usually learned design for harbor structures from their superior or their own. Because ofinefficient education way, every design firmhas their own design format and stores design data.Therefore, young engineers have difficult time understanding and learning the necessary design skills for harbor structures. So, the objects of this study is to improve current manual design process to become automated one to minimize errors from hand calculation, improve design speed, and allow various alternative design solutions. Ultimately, automated design process can lead to reducing bidding time and cost, accumulating optimum design-base, and decreasing construct cost. 


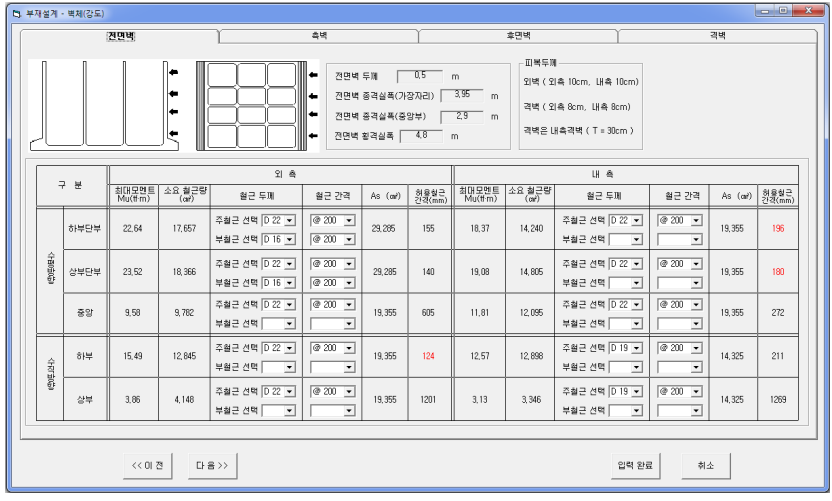

Fig.1 Member steel bar selection window

\section{COMPUTER AIDED TRAINING AND EDUCATION}

Currently, Korea Ocean Research \& Development Institute developed design software in 2003 in Korea, and Praia Consultant and ArataniCompany developed design program in Japan. However, the developed programs provide only input and output results to users, which were difficult to incorporate in design process, and without economical decision capability making it difficult to change modified design code. Comparing this software with previous design methodologies, this system can provide a standard design format that integrates various types of design plans.

The program is based on Window OS and using GUI (Graphic User Interface) so users can easily operate program. For user friendly interface, this program is written in MS Visual Basicwithaunified design format and for the output format, which is actual design calculation, is written in the MS Excel.This program display immediately drawing and calculated values corresponding to input values.Moreover, the input variablescan be saved out as a text file so that it may be modified for multiple design conditions and process.

Fig. 1 shows steel reinforcement selection window. Users are able to see instantly the moment value of members. Then, users can easily choose suitable number and size of steel reinforcements. If section area of chosen steel reinforcement or calculated crack width do not satisfy required steel area and crack width, the calculated values of section area and crack width would blink in red colors to notify the users. Fig. 2 shows quantity comparison window. Users are able to compare three different design types with respect to the weight of each material and price.Because users can

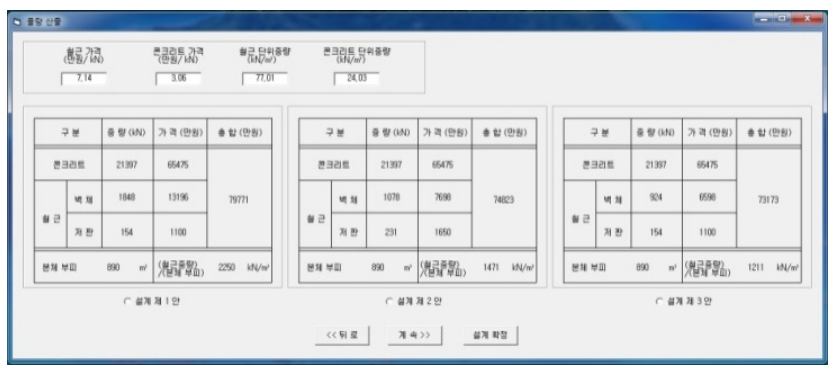

Fig.2 Quantities Comparison Window for 3 cases

input the market material price, users are able to choose effective design which satisfies price and safety.

\section{CONCLUSION}

Through the above process, using this program, young engineer can learn the whole process and examine closely details of design flow and design code. Especially, the output comes out excel file so that users can review whole design process and also details. Also, this system also helps young engineers to verify all input and output values where they can grasp theoverall design concepts and procedures. Ultimately, this program makes it possible to optimization of the total price.

\section{REFERENCES}

[1] Dym, C.L., "Engineering Design Thinking, Teaching, and Learning", Journal ofEngineering Education, Vol.94,No.1, 2005, pp.103-120.

[2] Dym, C. L., "Engineering Design: A ProjectBasedIntroduction", 2nd ed. John Wiley, New York, N.Y., 2003.

[3] Sheppard, S. D., "A Description of Engineering: An EssentialBackdrop for Interpreting Engineering Education", Proc. (CD),Mudd Design Workshop IV, Harvey Mudd College, Claremont, Cal. 